ANNALS OF

CLINICAL

NEUROPHYSIOLOGY
Received: August 3, 2020

Revised: August 25, 2020

Accepted: August 27, 2020

\section{Correspondence to}

\section{Kang Min Park}

Department of Neurology, Inje University Haeundae Paik Hospital, Inje University College of Medicine, 875 Haeun-daero, Haeundae-gu, Busan 48108, Korea Tel: +82-51-797-1195

Fax: +82-51-797-1196

E-mail:smilepkm@hanmail.net

* These authors equally contributed this work.

\section{ORCID}

\section{Bong Soo Park}

https://orcid.org/0000-0001-8999-386X Dongah Lee

https://orcid.org/0000-0001-8114-1371 Seongho Park

https://orcid.org/0000-0002-0504-2539 Kang Min Park

https://orcid.org/0000-0002-4229-7741

\title{
Which articles have highly impacted research on genetic generalized epilepsy?
}

\author{
Bong Soo Park ${ }^{1 *}$, Dongah Lee ${ }^{2 *}$, Seongho Park ${ }^{2}$, Kang Min Park ${ }^{2}$ \\ 'Department of Internal Medicine, Inje University Haeundae Paik Hospital, Inje University College of Medicine, \\ Busan, Korea \\ 2Department of Neurology, Inje University Haeundae Paik Hospital, Inje University College of Medicine, Busan, \\ Korea
}

Background: The purpose of this study was to identify the top-100 cited articles on genetic generalized epilepsy (GGE) published in journals that have made key contributions to the field of epilepsy.

Methods: We searched the Web of Science website produced by Clarivate Analytics for articles on GGE, and sorted them according to the number of citations to identify the top-100 cited articles. We then manually reviewed the contents of the top-100 cited articles, which were designated as "citation classics".

Results: The top-100 cited articles were published in 27 journals, with the largest proportion appearing in Epilepsia (19 articles). The articles originated from institutions in 17 countries, with 31 articles from the USA. The institution associated with the largest numbers of articles in the field of GGE was the University of Melbourne, Australia (9 articles). Panayiotopoulos C. P. was the first author of three articles, and was listed most frequently in the GGE citation classics. The publication years were concentrated in the 2000s, when 56 articles were published. The most-common study topics were genetics ( 35 articles) and neuroimaging (17 articles).

Conclusions: This study has identified the top-100 cited articles on GGE. These citation classics represent the landmark articles on GGE, and they provide useful insights into international research leaders and the research trends in the field.

Key words: Publication; Epilepsy, Idiopathic generalized; Retracted publication

\section{INTRODUCTION}

The International League Against Epilepsy (ILAE) classification of epilepsies was updated on 2017, and it now classifies epilepsy according to seizure type, epilepsy type, and etiology. ${ }^{1}$ 
Generalized epilepsy refers to the seizures originating at some point within or rapidly engaging distributed networks bilaterally, the subcortical or cortical structures, or frequently both of these. ' A genetic etiology is defined when epilepsy directly results from a known or presumed genetic defect and the seizures are the core symptom of the disorder. ${ }^{1}$ The term genetic generalized epilepsy (GGE) is used when a patient has generalized seizures of genetic origin and a well-recognized and established epilepsy syndrome, and includes childhood absence epilepsy, juvenile absence epilepsy, juvenile myoclonic epilepsy, and epilepsy with tonic-clonic seizures alone. ${ }^{1,2} \mathrm{GGE}$ has previously been termed "idiopathic generalized epilepsy," but the ILAE recommend changing this to "genetic generalized epilepsy" due to "idiopathic" being considered too imprecise. Individuals with GGE account for $20 \%$ of all epilepsy cases. ${ }^{3}$ GGE occurs mostly in young people, and with a proper diagnosis and management can be controlled with medications in $80 \%$ of cases. ${ }^{3}$

The number of times that a previously published work is cited is an indicator of its recognition and impact in an area of investigation. ${ }^{4-6}$ Citation analysis is a systematic approach for identifying scientific studies that have had a high impact in a particular field. Reviewing articles that are cited frequently can provide information about the dominant areas of a discipline, as well as identify growth areas in particular fields. Furthermore, the top-cited articles are often written by recognized experts who can offer novel insight into the future directions of the discipline. . $^{4-6}$

Several recent studies have applied citation analysis or bibliometric analysis to various neurological fields, including stroke, ${ }^{7}$ headache disorders, ${ }^{8}$ central nervous system inflammatory demyelinating disease, ${ }^{9}$ Guillain-Barré syndrome, ${ }^{10}$ epilepsy and status epilepticus, ${ }^{11}$ and general neurology. ${ }^{12}$ However, to the best of our knowledge, no previous study has comprehensively investigated the top-cited articles in the field of GGE. The purpose of this study was to identify the top-100 cited articles (designated as "citation classics") published in journals on GGE that have made key contributions to the field of epilepsy.

\section{MATERIALS AND METHODS}

A citation analysis is a bibliometric method that examines the frequency and patterns of citations in articles. We performed a citation analysis in the field of GGE by searching the Web of Science website (https://www.webofknowledge. com) produced by Clarivate Analytics.

In January 2020 we searched for articles published since 1950 with titles that included any of the following expressions: "genetic generalized epilepsy," "idiopathic generalized epilepsy," "childhood absence epilepsy," "juvenile absence epilepsy," "juvenile myoclonic epilepsy," "epilepsy with generalized tonic-clonic seizures alone," or "epilepsy with generalized tonic-clonic seizures on awakening." The top-100 cited articles were then selected according to the number of citations, and we manually reviewed their contents. We examined various aspects of the articles, such as the number of citations, ranking, authorship, title, year of publication, publishing journal, publication type, and topic categories. The publication types were categorized into original articles, case series, and systematic reviews, and the topics were subtyped as clinical features, epidemiology, pharmacotherapy, laboratory investigations, electrophysiology, neuroimaging, genetics, neuropsychiatry, and general reviews. When the authors of an article had more than one affiliation, the department, institution, and country of origin were defined by either the first or the corresponding affiliation of the first author. Data were presented using descriptive statistics, and no tests of statistical significance were performed. This study did not need to be reviewed by an ethics committee because it performed a bibliometric analysis of existing published studies.

\section{RESULTS}

We ranked the top-100 cited articles according to the number of citations (Table 1). The most-cited and least-cited articles had been cited 580 and 76 times, respectively. Approximately two-thirds of the articles (64 articles) had been cited more than 100 times.

The top-100 cited articles were published in 27 journals (Table 2), with the largest proportion appearing in Epilepsia (19 articles), followed by Neurology (15 articles) and Brain (10 articles). The top-100 cited articles originated from institutions in 17 countries, with 31 articles from the USA, followed by the UK (14 articles), Germany (11 articles), and 
Table 1. The top-100 cited articles in the field of GGE

\begin{tabular}{|c|c|c|c|c|c|c|c|c|}
\hline Rank & Title & First author & Journal & Year & $\begin{array}{l}\text { Vol- } \\
\text { ume }\end{array}$ & $\begin{array}{l}\text { First } \\
\text { page }\end{array}$ & $\begin{array}{l}\text { Last } \\
\text { page }\end{array}$ & $\begin{array}{l}\text { Number of } \\
\text { citations }\end{array}$ \\
\hline 1 & $\begin{array}{l}\text { Mutant GABA(A) receptor gamma 2-subunit in child- } \\
\text { hood absence epilepsy and febrile seizures }\end{array}$ & Wallace $\mathrm{RH}$ & Nature Genetics & 2001 & 28 & 49 & 52 & 580 \\
\hline 2 & $\begin{array}{l}\text { Mutation of GABRA1 in an autosomal dominant form of } \\
\text { juvenile myoclonic epilepsy }\end{array}$ & Cossette P & Nature Genetics & 2002 & 31 & 184 & 189 & 403 \\
\hline 3 & $\begin{array}{l}\text { Childhood absence epilepsy: genes, channels, neurons } \\
\text { and networks }\end{array}$ & Crunelli V & $\begin{array}{l}\text { Nature Reviews Neurosci- } \\
\text { ence }\end{array}$ & 2002 & 3 & 371 & 382 & 397 \\
\hline 4 & $\begin{array}{l}\text { 15q13.3 microdeletions increase risk of idiopathic gener- } \\
\text { alized epilepsy }\end{array}$ & Helbig I & Nature Genetics & 2009 & 41 & 160 & 162 & 393 \\
\hline 5 & Juvenile myoclonic epilepsy of Janz & $\begin{array}{l}\text { Delgadoes- } \\
\text { cueta AV }\end{array}$ & Neurology & 1984 & 34 & 285 & 294 & 310 \\
\hline 6 & $\begin{array}{l}\text { Genome-wide copy number variation in epilepsy: } \\
\text { novel susceptibility loci in idiopathic generalized and } \\
\text { focal epilepsies }\end{array}$ & Mefford HC & PLOS Genetics & 2010 & 6 & 1 & 9 & 308 \\
\hline 7 & $\begin{array}{l}\text { Recurrent microdeletions at 15q11.2 and 16p13.11 predis- } \\
\text { pose to idiopathic generalized epilepsies }\end{array}$ & de Kovel CG & Brain & 2010 & 133 & 23 & 32 & 293 \\
\hline 8 & $\begin{array}{l}\text { Juvenile myoclonic epilepsy (JME) may be linked to the } \\
\text { BF and HLA loci on human chromosome } 6\end{array}$ & $\begin{array}{l}\text { Greenberg } \\
\text { DA }\end{array}$ & $\begin{array}{l}\text { American Journal of Medi- } \\
\text { cal Genetics }\end{array}$ & 1988 & 31 & 185 & 192 & 285 \\
\hline 9 & $\begin{array}{l}\text { Altered functional-structural coupling of large-scale } \\
\text { brain networks in idiopathic generalized epilepsy }\end{array}$ & Zhang Z & Brain & 2011 & 134 & 2912 & 2928 & 267 \\
\hline 10 & $\begin{array}{l}\text { Ethosuximide, valproic acid, and lamotrigine in child- } \\
\text { hood absence epilepsy }\end{array}$ & Glauser TA & $\begin{array}{l}\text { New England Journal of } \\
\text { Medicine }\end{array}$ & 2010 & 362 & 790 & 799 & 262 \\
\hline 11 & $\begin{array}{l}\text { fMRI activation during spike and wave discharges in } \\
\text { idiopathic generalized epilepsy }\end{array}$ & Aghakhani Y & Brain & 2004 & 127 & 1127 & 1144 & 260 \\
\hline 12 & $\begin{array}{l}\text { Mutations in CLCN2 encoding a voltage-gated chloride } \\
\text { channel are associated with idiopathic generalized } \\
\text { epilepsies (retracted article. See vol 41, pg. 1043, 2009) }\end{array}$ & Haug K & Nature Genetics & 2003 & 33 & 527 & 532 & 251 \\
\hline 13 & $\begin{array}{l}\text { Coding and noncoding variation of the human calci- } \\
\text { um-channel beta(4)-subunit gene CACNB4 in patients } \\
\text { with idiopathic generalized epilepsy and episodic } \\
\text { ataxia }\end{array}$ & Escayg A & $\begin{array}{l}\text { American Journal of Medi- } \\
\text { cal Genetics }\end{array}$ & 2000 & 66 & 1531 & 1539 & 248 \\
\hline 14 & $\begin{array}{l}\text { Association between genetic variation of } \mathrm{CACNA1H} \\
\text { and childhood absence epilepsy }\end{array}$ & Chen YC & Annals of Neurology & 2003 & 54 & 239 & 243 & 246 \\
\hline 15 & $\begin{array}{l}\text { Abnormal cerebral structure in juvenile myoclonic epi- } \\
\text { lepsy demonstrated with voxel-based analysis of MRI }\end{array}$ & $\begin{array}{l}\text { Woermann } \\
\quad \text { FG }\end{array}$ & Brain & 1999 & 122 & 2101 & 2107 & 235 \\
\hline 15 & $\begin{array}{l}\text { Epilepsy with impulsive petit mal (juvenile myoclonic } \\
\text { epilepsy) }\end{array}$ & Janz D & $\begin{array}{l}\text { Acta Neurologica Scandi- } \\
\text { navica }\end{array}$ & 1985 & 72 & 449 & 459 & 235 \\
\hline 17 & Mutations in EFHC1 cause juvenile myoclonic epilepsy & Suzuki T & Nature Genetics & 2004 & 36 & 842 & 849 & 229 \\
\hline 18 & Juvenile myoclonic epilepsy: a 5-year prospective study & $\begin{array}{l}\text { Panayio- } \\
\text { topoulos CP }\end{array}$ & Epilepsia & 1994 & 35 & 285 & 296 & 225 \\
\hline 19 & $\begin{array}{l}\text { Genetic mapping of a major susceptibility locus for } \\
\text { juvenile myoclonic epilepsy on chromosome } 15 q\end{array}$ & Elmslie FV & $\begin{array}{l}\text { Human Molecular Genet- } \\
\text { ics }\end{array}$ & 1997 & 6 & 1329 & 1334 & 217 \\
\hline 20 & $\begin{array}{l}\text { A splice-site mutation in GABRG2 associated with child- } \\
\text { hood absence epilepsy and febrile convulsions }\end{array}$ & Kananura C & Archives of Neurology & 2002 & 59 & 1137 & 1141 & 194 \\
\hline
\end{tabular}


Table 1. Continued

\begin{tabular}{|c|c|c|c|c|c|c|c|c|}
\hline Rank & Title & First author & Journal & Year & $\begin{array}{l}\text { Vol- } \\
\text { ume }\end{array}$ & $\begin{array}{l}\text { First } \\
\text { page }\end{array}$ & $\begin{array}{l}\text { Last } \\
\text { page }\end{array}$ & $\begin{array}{l}\text { Number of } \\
\text { citations }\end{array}$ \\
\hline 21 & $\begin{array}{l}\text { Localization of idiopathic generalized epilepsy on chro- } \\
\text { mosome } 6 p \text { in families of juvenile myoclonic epilepsy } \\
\text { patients }\end{array}$ & Durner M & Neurology & 1991 & 41 & 1651 & 1655 & 189 \\
\hline 22 & $\begin{array}{l}\text { Childhood absence epilepsy: behavioral, cognitive, and } \\
\text { linguistic comorbidities }\end{array}$ & Caplan R & Epilepsia & 2008 & 49 & 1838 & 1846 & 185 \\
\hline 23 & $\begin{array}{l}\text { EEG-fMRI of idiopathic and secondarily generalized epi- } \\
\text { lepsies }\end{array}$ & Hamandi K & Neurolmage & 2006 & 31 & 1700 & 1710 & 179 \\
\hline 24 & $\begin{array}{l}\text { Interictal mood and personality disorders in temporal } \\
\text { lobe epilepsy and juvenile myoclonic epilepsy }\end{array}$ & Perini Gl & $\begin{array}{l}\text { Journal of Neurology Neu- } \\
\text { rosurgery and Psychiatry }\end{array}$ & 1996 & 61 & 601 & 605 & 170 \\
\hline 25 & $\begin{array}{l}\text { Familial and sporadic } 15 q 13.3 \text { microdeletions in idio- } \\
\text { pathic generalized epilepsy: precedent for disorders } \\
\text { with complex inheritance }\end{array}$ & Dibbens LM & $\begin{array}{l}\text { Human Molecular Genet- } \\
\text { ics }\end{array}$ & 2009 & 18 & 3626 & 3631 & 164 \\
\hline 26 & $\begin{array}{l}\text { Confirmation of linkage between juvenile myoclonic } \\
\text { epilepsy locus and the HLA region of chromosome } 6\end{array}$ & $\begin{array}{l}\text { Weissbecker } \\
\text { KA }\end{array}$ & $\begin{array}{l}\text { American Journal of Medi- } \\
\text { cal Genetics }\end{array}$ & 1991 & 38 & 32 & 36 & 160 \\
\hline 27 & $\begin{array}{l}\text { Placebo-controlled study of levetiracetam in idiopathic } \\
\text { generalized epilepsy }\end{array}$ & Berkovic SF & Neurology & 2007 & 69 & 1751 & 1760 & 155 \\
\hline 28 & $\begin{array}{l}\text { Levetiracetam for the treatment of idiopathic general- } \\
\text { ized epilepsy with myoclonic seizures }\end{array}$ & Noachtar S & Neurology & 2008 & 70 & 607 & 616 & 153 \\
\hline 28 & Epidemiology of idiopathic generalized epilepsies & Jallon P & Epilepsia & 2005 & 46 & 10 & 14 & 153 \\
\hline 28 & $\begin{array}{l}\text { Long-term prognosis in two forms of childhood ep- } \\
\text { ilepsy: typical absence seizures and epilepsy with } \\
\text { rolandic (centrotemporal) EEG foci }\end{array}$ & Loiseau P & Annals of Neurology & 1983 & 13 & 642 & 648 & 153 \\
\hline 31 & $\begin{array}{l}\text { Genome search for susceptibility loci of common idio- } \\
\text { pathic generalised epilepsies }\end{array}$ & Sander $T$ & $\begin{array}{l}\text { Human Molecular Genet- } \\
\text { ics }\end{array}$ & 2000 & 9 & 1465 & 1472 & 140 \\
\hline 32 & $\begin{array}{l}\text { Reduced cortical inhibition in a mouse model of familial } \\
\text { childhood absence epilepsy }\end{array}$ & Tan $\mathrm{HO}$ & $\begin{array}{l}\text { Proceedings of the } \\
\text { National Academy of Sci- } \\
\text { ences of the United States } \\
\text { of America }\end{array}$ & 2007 & 104 & 17536 & 17541 & 136 \\
\hline 33 & $\begin{array}{l}\text { Absence and myoclonic status epilepticus precipitated } \\
\text { by antiepileptic drugs in idiopathic generalized epilepsy }\end{array}$ & Thomas P & Brain & 2006 & 129 & 1281 & 1292 & 134 \\
\hline 34 & $\begin{array}{l}\text { MRI volumetry of the thalamus in temporal, extratem- } \\
\text { poral, and idiopathic generalized epilepsy }\end{array}$ & Natsume J & Neurology & 2003 & 60 & 1296 & 1300 & 129 \\
\hline 34 & $\begin{array}{l}\text { Some clinical and EEG aspects of benign juvenile my- } \\
\text { oclonic epilepsy }\end{array}$ & Asconape J & Epilepsia & 1984 & 25 & 108 & 114 & 129 \\
\hline 36 & $\begin{array}{l}\text { Functional characterization and neuronal modeling of } \\
\text { the effects of childhood absence epilepsy variants of } \\
\text { CACNAIH, a T-type calcium channel }\end{array}$ & Vitko I & Journal of Neuroscience & 2005 & 25 & 4844 & 4855 & 128 \\
\hline 36 & Frontal functions in juvenile myoclonic epilepsy & Devinsky $O$ & $\begin{array}{l}\text { Neuropsychiatry Neuro- } \\
\text { psychology and Behavior- } \\
\text { al Neurology }\end{array}$ & 1997 & 10 & 243 & 246 & 128 \\
\hline 38 & $\begin{array}{l}\text { Mapping of spontaneous spike and wave discharges in } \\
\text { Wistar rats with genetic generalized nonconvulsive } \\
\text { epilepsy }\end{array}$ & Vergnes M & Brain Research & 1990 & 523 & 87 & 91 & 127 \\
\hline
\end{tabular}


Table 1. Continued

\begin{tabular}{|c|c|c|c|c|c|c|c|c|}
\hline Rank & Title & First author & Journal & Year & $\begin{array}{l}\text { Vol- } \\
\text { ume }\end{array}$ & $\begin{array}{l}\text { First } \\
\text { page }\end{array}$ & $\begin{array}{l}\text { Last } \\
\text { page }\end{array}$ & $\begin{array}{l}\text { Number of } \\
\text { citations }\end{array}$ \\
\hline 39 & $\begin{array}{l}\text { MR spectroscopy shows reduced frontal lobe concen- } \\
\text { trations of N-acetyl aspartate in patients with juvenile } \\
\text { myoclonic epilepsy }\end{array}$ & Savic I & Epilepsia & 2000 & 41 & 290 & 296 & 126 \\
\hline 40 & $\begin{array}{l}\text { Genome scan of idiopathic generalized epilepsy: evi- } \\
\text { dence for major susceptibility gene and modifying } \\
\text { genes influencing the seizure type }\end{array}$ & Durner M & Annals of Neurology & 2001 & 49 & 328 & 335 & 125 \\
\hline 41 & $\begin{array}{l}\text { Voltage-gated calcium channels and idiopathic gener- } \\
\text { alized epilepsies }\end{array}$ & Khosravani H & Physiological Reviews & 2006 & 86 & 941 & 966 & 124 \\
\hline 42 & $\begin{array}{l}\text { Do carbamazepine and phenytoin aggravate juvenile } \\
\text { myoclonic epilepsy? }\end{array}$ & Genton P & Neurology & 2000 & 55 & 1106 & 1109 & 123 \\
\hline 42 & $\begin{array}{l}\text { Long-term prognosis of typical childhood absence ep- } \\
\text { ilepsy: remission or progression to juvenile myoclonic } \\
\text { epilepsy }\end{array}$ & Wirrell EC & Neurology & 1996 & 47 & 912 & 918 & 123 \\
\hline 44 & $\begin{array}{l}\text { Gating effects of mutations in the Ca(v)3.2 T-type } \\
\text { calcium channel associated with childhood absence } \\
\text { epilepsy }\end{array}$ & Khosravani H & $\begin{array}{l}\text { Journal of Biological } \\
\text { Chemistry }\end{array}$ & 2004 & 279 & 9681 & 9684 & 121 \\
\hline 45 & $\begin{array}{l}\text { Ethosuximide, valproic acid, and lamotrigine in child- } \\
\text { hood absence epilepsy: initial monotherapy out- } \\
\text { comes at } 12 \text { months }\end{array}$ & Glauser TA & Epilepsia & 2013 & 54 & 141 & 155 & 119 \\
\hline 46 & $\begin{array}{l}\text { Primary (idiopathic) generalized epilepsy and underly- } \\
\text { ing mechanisms }\end{array}$ & $\begin{array}{l}\text { Niedermeyer } \\
\text { E }\end{array}$ & $\begin{array}{l}\text { Clinical Electroencepha- } \\
\text { lography }\end{array}$ & 1996 & 27 & 1 & 21 & 118 \\
\hline 47 & $\begin{array}{l}\text { Juvenile myoclonic epilepsy } 25 \text { years after seizure on- } \\
\text { set: a population-based study }\end{array}$ & Camfield CS & Neurology & 2009 & 73 & 1041 & 1045 & 117 \\
\hline 47 & $\begin{array}{l}\text { Elevated anxiety and depressive-like behavior in a rat } \\
\text { model of genetic generalized epilepsy suggesting } \\
\text { common causation }\end{array}$ & Jones NC & Experimental Neurology & 2008 & 209 & 254 & 260 & 117 \\
\hline 49 & $\begin{array}{l}\text { Quantitative MRI in patients with idiopathic generalized } \\
\text { epilepsy. Evidence of widespread cerebral structural } \\
\text { changes }\end{array}$ & $\begin{array}{l}\text { Woermann } \\
\qquad \text { FG }\end{array}$ & Brain & 1998 & 121 & 1661 & 1667 & 116 \\
\hline 49 & $\begin{array}{l}\text { Juvenile myoclonic epilepsy locus in chromosome } \\
\text { 6p21.2-p11: linkage to convulsions and electroenceph- } \\
\text { alography trait }\end{array}$ & Liu AW & $\begin{array}{l}\text { American Journal of } \\
\text { Human Genetics }\end{array}$ & 1995 & 57 & 368 & 381 & 116 \\
\hline 51 & $\begin{array}{l}\text { Extended spectrum of idiopathic generalized epilep- } \\
\text { sies associated with CACNA1H functional variants }\end{array}$ & Heron SE & Annals of Neurology & 2007 & 62 & 560 & 568 & 115 \\
\hline 52 & $\begin{array}{l}\text { Motor system hyperconnectivity in juvenile myoclonic } \\
\text { epilepsy: a cognitive functional magnetic resonance } \\
\text { imaging study }\end{array}$ & Vollmar C & Brain & 2011 & 134 & 1710 & 1719 & 114 \\
\hline 52 & $\begin{array}{l}\text { Voxel-based morphometry in patients with idiopathic } \\
\text { generalized epilepsies }\end{array}$ & Betting LE & Neurolmage & 2006 & 32 & 498 & 502 & 114 \\
\hline 52 & $\begin{array}{l}\text { Mapping of genes predisposing to idiopathic general- } \\
\text { ized epilepsy }\end{array}$ & Zara F & $\begin{array}{l}\text { Human Molecular Genet- } \\
\text { ics }\end{array}$ & 1995 & 4 & 1201 & 1207 & 114 \\
\hline 55 & $\begin{array}{l}\text { BRD2 (RING3) is a probable major susceptibility gene } \\
\text { for common juvenile myoclonic epilepsy }\end{array}$ & Pal DK & $\begin{array}{c}\text { American Journal of } \\
\text { Human Genetics }\end{array}$ & 2003 & 73 & 261 & 270 & 113 \\
\hline
\end{tabular}


Table 1. Continued

\begin{tabular}{|c|c|c|c|c|c|c|c|c|}
\hline Rank & Title & First author & Journal & Year & $\begin{array}{l}\text { Vol- } \\
\text { ume }\end{array}$ & $\begin{array}{l}\text { First } \\
\text { page }\end{array}$ & $\begin{array}{l}\text { Last } \\
\text { page }\end{array}$ & $\begin{array}{l}\text { Number of } \\
\text { citations }\end{array}$ \\
\hline 56 & $\begin{array}{l}\text { From molecules to networks: cortical/subcortical inter- } \\
\text { actions in the pathophysiology of idiopathic general- } \\
\text { ized epilepsy }\end{array}$ & $\begin{array}{c}\text { Blumenfeld } \\
\mathrm{H}\end{array}$ & Epilepsia & 2003 & 44 & 7 & 15 & 109 \\
\hline 57 & $\begin{array}{l}\text { Cognitive function in idiopathic generalized epilepsy of } \\
\text { childhood }\end{array}$ & Henkin Y & $\begin{array}{l}\text { Developmental Medicine } \\
\text { and Child Neurology }\end{array}$ & 2005 & 47 & 126 & 132 & 107 \\
\hline 58 & $\begin{array}{l}\text { Focal structural changes and cognitive dysfunction in } \\
\text { juvenile myoclonic epilepsy }\end{array}$ & $\begin{array}{c}\text { O'Muir- } \\
\text { cheartaigh J }\end{array}$ & Neurology & 2011 & 76 & 34 & 40 & 106 \\
\hline 58 & $\begin{array}{l}\text { Childhood absence epilepsy and febrile seizures: a fam- } \\
\text { ily with a GABA(A) receptor mutation }\end{array}$ & Marini C & Brain & 2003 & 126 & 230 & 240 & 106 \\
\hline 60 & $\begin{array}{l}\text { Hyperglycosylation and reduced GABA currents of } \\
\text { mutated GABRB3 polypeptide in remitting childhood } \\
\text { absence epilepsy }\end{array}$ & Tanaka M & $\begin{array}{l}\text { American Journal of } \\
\text { Human Genetics }\end{array}$ & 2008 & 82 & 1249 & 1261 & 104 \\
\hline 60 & $\begin{array}{l}\text { Reproducibility and complications in gene searches: } \\
\text { linkage on chromosome } 6 \text {, heterogeneity, associa- } \\
\text { tion, and maternal inheritance in juvenile myoclonic } \\
\text { epilepsy }\end{array}$ & $\begin{array}{l}\text { Greenberg } \\
\text { DA }\end{array}$ & $\begin{array}{l}\text { American Journal of } \\
\text { Human Genetics }\end{array}$ & 2000 & 66 & 508 & 516 & 104 \\
\hline 62 & $\begin{array}{l}\text { Clinical factors of drug resistance in juvenile myoclonic } \\
\text { epilepsy }\end{array}$ & Gelisse P & $\begin{array}{l}\text { Journal of Neurology Neu- } \\
\text { rosurgery and Psychiatry }\end{array}$ & 2001 & 70 & 240 & 243 & 102 \\
\hline 63 & $\begin{array}{l}\text { Thalamofrontal circuitry and executive dysfunction in } \\
\text { recent-onset juvenile myoclonic epilepsy }\end{array}$ & Pulsipher DT & Epilepsia & 2009 & 50 & 1210 & 1219 & 100 \\
\hline 63 & $\begin{array}{l}\text { Neuropsychological profile of patients with juvenile } \\
\text { myoclonic epilepsy: a controlled study of } 50 \text { patients }\end{array}$ & $\begin{array}{l}\text { Pascalicchio } \\
\text { TF }\end{array}$ & Epilepsy and Behavior & 2007 & 10 & 263 & 267 & 100 \\
\hline 65 & $\begin{array}{l}\text { Genome-wide association analysis of genetic general- } \\
\text { ized epilepsies implicates susceptibility loci at 1q43, } \\
\text { 2p16.1, 2q22.3 and 17q21.32 }\end{array}$ & Steffens M & $\begin{array}{l}\text { Human Molecular Genet- } \\
\text { ics }\end{array}$ & 2012 & 21 & 5359 & 5372 & 98 \\
\hline 66 & $\begin{array}{l}\text { Childhood absence epilepsy with tonic-clonic seizures } \\
\text { and electroencephalogram 3-4-Hz spike and multis- } \\
\text { pike-slow wave complexes: linkage to chromosome } \\
\text { 8q24 }\end{array}$ & Fong GCY & $\begin{array}{l}\text { American Journal of } \\
\text { Human Genetics }\end{array}$ & 1998 & 63 & 1117 & 1129 & 97 \\
\hline 66 & $\begin{array}{l}\text { Linkage analysis of idiopathic generalized epilepsy (IGE) } \\
\text { and marker loci on chromosome-6p in families of pa- } \\
\text { tients with juvenile myoclonic epilepsy: no evidence } \\
\text { for an epilepsy locus in the HLA region }\end{array}$ & $\begin{array}{l}\text { Whitehouse } \\
\text { WP }\end{array}$ & $\begin{array}{l}\text { American Journal of } \\
\text { Human Genetics }\end{array}$ & 1993 & 53 & 652 & 662 & 97 \\
\hline 66 & $\begin{array}{l}\text { Juvenile myoclonic epilepsy: factors of error involved in } \\
\text { the diagnosis and treatment }\end{array}$ & $\begin{array}{l}\text { Panayio- } \\
\text { topoulos CP }\end{array}$ & Epilepsia & 1991 & 32 & 672 & 676 & 97 \\
\hline 69 & $\begin{array}{l}\text { Clinical and EEG asymmetries in juvenile myoclonic epi- } \\
\text { lepsy }\end{array}$ & Lancman ME & Epilepsia & 1994 & 35 & 302 & 306 & 96 \\
\hline 69 & $\begin{array}{l}\text { Juvenile myoclonic epilepsy: long-term response to } \\
\text { therapy }\end{array}$ & Penry JK & Epilepsia & 1989 & 30 & S19 & $\mathrm{S} 23$ & 96 \\
\hline 71 & $\begin{array}{l}\text { Perampanel for tonic-clonic seizures in idiopathic gen- } \\
\text { eralized epilepsy. A randomized trial }\end{array}$ & French JA & Neurology & 2015 & 85 & 950 & 957 & 95 \\
\hline 71 & $\begin{array}{l}\text { Nerve fiber impairment of anterior thalamocortical cir- } \\
\text { cuitry in juvenile myoclonic epilepsy }\end{array}$ & Deppe M & Neurology & 2008 & 71 & 1981 & 1985 & 95 \\
\hline
\end{tabular}


Table 1. Continued

\begin{tabular}{|c|c|c|c|c|c|c|c|c|}
\hline Rank & Title & First author & Journal & Year & $\begin{array}{l}\text { Vol- } \\
\text { ume }\end{array}$ & $\begin{array}{l}\text { First } \\
\text { page }\end{array}$ & $\begin{array}{l}\text { Last } \\
\text { page }\end{array}$ & $\begin{array}{l}\text { Number of } \\
\text { citations }\end{array}$ \\
\hline 73 & $\begin{array}{l}\text { Regional grey matter abnormalities in juvenile myoc- } \\
\text { lonic epilepsy: a voxel-based morphometry study }\end{array}$ & Kim JH & Neurolmage & 2007 & 37 & 1132 & 1137 & 93 \\
\hline 73 & $\begin{array}{l}\text { The GABA(A) receptor gamma } 2 \text { subunit R43Q muta- } \\
\text { tion linked to childhood absence epilepsy and febrile } \\
\text { seizures causes retention of alpha } 1 \text { beta } 2 \text { gamma } 2 S \\
\text { receptors in the endoplasmic reticulum }\end{array}$ & Kang JQ & Journal of Neuroscience & 2004 & 24 & 8672 & 8677 & 93 \\
\hline 73 & $\begin{array}{l}\text { Magnetic resonance spectroscopy and imaging of the } \\
\text { thalamus in idiopathic generalized epilepsy }\end{array}$ & Bernasconi A & Brain & 2003 & 126 & 2447 & 2454 & 93 \\
\hline 76 & $\begin{array}{l}\text { Impaired attention and network connectivity in child- } \\
\text { hood absence epilepsy }\end{array}$ & Killory BD & Neurolmage & 2011 & 56 & 2209 & 2217 & 92 \\
\hline 76 & $\begin{array}{l}\text { The relationship between treatment with valproate, } \\
\text { lamotrigine, and topiramate and the prognosis of the } \\
\text { idiopathic generalised epilepsies }\end{array}$ & Nicolson A & $\begin{array}{l}\text { Journal of Neurology Neu- } \\
\text { rosurgery and Psychiatry }\end{array}$ & 2004 & 75 & 75 & 79 & 92 \\
\hline 78 & $\begin{array}{l}\text { Thalamo-cortical network pathology in idiopathic gen- } \\
\text { eralized epilepsy: insights from MRI-based morpho- } \\
\text { metric correlation analysis }\end{array}$ & Bernhardt BC & Neurolmage & 2009 & 46 & 373 & 381 & 91 \\
\hline 78 & $\begin{array}{l}\text { Why does fever trigger febrile seizures? GABA(A) recep- } \\
\text { tor gamma } 2 \text { subunit mutations associated with idio- } \\
\text { pathic generalized epilepsies have temperature-de- } \\
\text { pendent trafficking deficiencies }\end{array}$ & Kang JQ & Journal of Neuroscience & 2006 & 26 & 2590 & 2597 & 91 \\
\hline 78 & $\begin{array}{l}\text { Focal electroencephalographic abnormalities in juve- } \\
\text { nile myoclonic epilepsy }\end{array}$ & Aliberti V & Epilepsia & 1994 & 35 & 297 & 301 & 91 \\
\hline 78 & Juvenile myoclonic epilepsy: a study in Saudi Arabia & Obeid T & Epilepsia & 1988 & 29 & 280 & 282 & 91 \\
\hline 82 & $\begin{array}{l}\text { Pretreatment cognitive deficits and treatment effects } \\
\text { on attention in childhood absence epilepsy }\end{array}$ & Masur D & Neurology & 2013 & 81 & 1572 & 1580 & 88 \\
\hline 82 & $\begin{array}{l}\text { Electroclinical features of absence seizures in childhood } \\
\text { absence epilepsy }\end{array}$ & Sadleir LG & Neurology & 2006 & 67 & 413 & 418 & 88 \\
\hline 82 & $\begin{array}{l}\text { Genetic architecture of idiopathic generalized epilepsy: } \\
\text { clinical genetic analysis of } 55 \text { multiplex families }\end{array}$ & Marini C & Epilepsia & 2004 & 45 & 467 & 478 & 88 \\
\hline 82 & Juvenile myoclonic epilepsy. A review & $\begin{array}{l}\text { Grunewald } \\
\text { RA }\end{array}$ & Archives of Neurology & 1993 & 50 & 594 & 598 & 88 \\
\hline 86 & $\begin{array}{l}\text { Juvenile myoclonic epilepsy subsyndromes: family } \\
\text { studies and long-term follow-up }\end{array}$ & $\begin{array}{c}\text { Marti- } \\
\text { nez-Juarez IE }\end{array}$ & Brain & 2006 & 129 & 1269 & 1280 & 86 \\
\hline 87 & $\begin{array}{l}\text { Proton MRS reveals frontal lobe metabolite abnormali- } \\
\text { ties in idiopathic generalized epilepsy }\end{array}$ & Simister RJ & Neurology & 2003 & 61 & 897 & 902 & 85 \\
\hline 88 & $\begin{array}{l}\text { Exacerbation of juvenile myoclonic epilepsy with lamo- } \\
\text { trigine }\end{array}$ & Biraben A & Neurology & 2000 & 55 & 1758 & 1758 & 84 \\
\hline 89 & $\begin{array}{l}\text { Multi-site voxel-based morphometry: methods and } \\
\text { a feasibility demonstration with childhood absence } \\
\text { epilepsy }\end{array}$ & Pardoe H & Neurolmage & 2008 & 42 & 611 & 616 & 83 \\
\hline 89 & Delayed diagnosis of juvenile myoclonic epilepsy & $\begin{array}{l}\text { Grunewald } \\
\text { RA }\end{array}$ & $\begin{array}{l}\text { Journal of Neurology Neu- } \\
\text { rosurgery and Psychiatry }\end{array}$ & 1992 & 55 & 497 & 499 & 83 \\
\hline
\end{tabular}


Table 1. Continued

\begin{tabular}{|c|c|c|c|c|c|c|c|c|}
\hline Rank & Title & First author & Journal & Year & $\begin{array}{l}\text { Vol- } \\
\text { ume }\end{array}$ & $\begin{array}{l}\text { First } \\
\text { page }\end{array}$ & $\begin{array}{l}\text { Last } \\
\text { page }\end{array}$ & $\begin{array}{l}\text { Number of } \\
\text { citations }\end{array}$ \\
\hline 91 & $\begin{array}{l}\text { The idiopathic generalized epilepsies of adolescence } \\
\text { with childhood and juvenile age of onset }\end{array}$ & Janz D & Epilepsia & 1997 & 38 & 4 & 11 & 82 \\
\hline 92 & $\begin{array}{l}\text { Glucose transporter } 1 \text { deficiency in the idiopathic gen- } \\
\text { eralized epilepsies }\end{array}$ & Arsov $T$ & Annals of Neurology & 2012 & 72 & 807 & 815 & 81 \\
\hline 93 & $\begin{array}{l}\text { Idiopathic generalized epilepsies recognized by the } \\
\text { International League Against Epilepsy }\end{array}$ & Nordli DR & Epilepsia & 2005 & 46 & 48 & 56 & 80 \\
\hline 94 & $\begin{array}{l}\text { Genome arrays for the detection of copy number } \\
\text { variations in idiopathic mental retardation, idiopathic } \\
\text { generalized epilepsy and neuropsychiatric disorders: } \\
\text { lessons for diagnostic workflow and research }\end{array}$ & $\begin{array}{l}\text { Hochsten- } \\
\text { bach } R\end{array}$ & $\begin{array}{l}\text { Cytogenetic and Genome } \\
\text { Research }\end{array}$ & 2011 & 135 & 174 & 202 & 79 \\
\hline 94 & $\begin{array}{l}\text { The I-II loop controls plasma membrane expression and } \\
\text { gating of Ca(v)3.2 T-type Ca2+ channels: a paradigm } \\
\text { for childhood absence epilepsy mutations }\end{array}$ & Vitko I & Journal of Neuroscience & 2007 & 27 & 322 & 330 & 79 \\
\hline 96 & $\begin{array}{l}\text { Sleep microstructure and EEG epileptiform activity in } \\
\text { patients with juvenile myoclonic epilepsy }\end{array}$ & Gigli GL & Epilepsia & 1992 & 33 & 799 & 804 & 78 \\
\hline 96 & $\begin{array}{l}\text { Juvenile myoclonic epilepsy: an autosomal recessive } \\
\text { disease }\end{array}$ & $\begin{array}{l}\text { Panayio- } \\
\text { topoulos CP }\end{array}$ & Annals of Neurology & 1989 & 25 & 440 & 443 & 78 \\
\hline 98 & Thalamic atrophy in childhood absence epilepsy & Chan $\mathrm{CH}$ & Epilepsia & 2006 & 47 & 399 & 405 & 76 \\
\hline 98 & $\begin{array}{l}\text { Worsening of seizures by oxcarbazepine in juvenile } \\
\text { idiopathic generalized epilepsies }\end{array}$ & Gelisse P & Epilepsia & 2004 & 45 & 1282 & 1286 & 76 \\
\hline 98 & $\begin{array}{l}\text { Tiagabine-induced absence status in idiopathic gener- } \\
\text { alized epilepsy }\end{array}$ & Knake S & $\begin{array}{l}\text { Seizure - European Journal } \\
\text { of Epilepsy }\end{array}$ & 1999 & 8 & 314 & 317 & 76 \\
\hline
\end{tabular}

GGE, genetic generalized epilepsy; GABA, gamma-Aminobutyric acid; JME, Juvenile myoclonic epilepsy; Bf, factor B; HLA, human leukocyte antigen; IGE, idiopathic generalized epilepsy; fMRI, functional magnetic resonance imaging; CLCN2, chloride voltage-gated channel 2; CACNB4, calcium voltage-gated channel auxiliary subunit beta 4; EEFHC1, EF-hand domain containing 1; GABRG2, gamma-Aminobutyric Acid type A receptor subunit gamma 2; EEG, electroencephalography; MRI, magnetic resonance imaging; RING3, Really Interesting New Gene 3; MRS, MR spectroscopy.

Australia (11 articles) (Table 3). The 100 articles comprised 40 originating from North America (the USA and Canada), 38 from Europe (the UK, Germany, France, Italy, Netherlands, Switzerland, and Sweden), 12 from Oceania (Australia and New Zealand), 8 from Asia (Saudi Arabia, China, South Korea, Israel, and Japan), and 2 from South America (Brazil).

Tables 4 and 5 list the top-ranked institutions and authors for articles published in the field of GGE, respectively. The institution associated with the largest number of articles was the University of Melbourne, Australia (nine articles), followed by the University of California in Los Angeles, USA (seven articles), and University College London, UK (six articles). Panayiotopoulos C. P. was the first author of three articles, and was listed most frequently in the GGE citation classics.
The publication years were mostly concentrated in the 2000s, when 56 articles were published. Twenty-three articles were published in the 1990s, followed by 13 articles in the 2010s, and 8 in the 1980s. The earliest recorded article was published in 1983 and the most-recent article was published in 2015.

Regarding the types of articles, 95 were original articles while 5 were systematic review articles. The subjects of the articles comprised 22 on childhood absence epilepsy, 38 on juvenile myoclonic epilepsy, and 40 on GGE as a whole. The topic subtypes of the articles comprised 35 on genetics, 17 on neuroimaging, 13 on pharmacotherapy, 13 on electrophysiology, 8 on neuropsychiatry, 5 on epidemiology, 5 on general reviews, and 4 on clinical features (Fig. 1). 
Table 2. Journals containing at least 2 of the top-100 cited articles in the field of GGE

\begin{tabular}{lcc}
\hline Rank & Journal & $\begin{array}{c}\text { Number } \\
\text { of articles }\end{array}$ \\
\hline 1 & Epilepsia & 19 \\
\hline 2 & Neurology & 15 \\
\hline 3 & Brain & 10 \\
\hline 4 & American Journal of Human Genetics & 6 \\
\hline 4 & Annals of Neurology & 6 \\
\hline 4 & Neurolmage & 6 \\
\hline 7 & Human Molecular Genetics & 5 \\
\hline 7 & Nature Genetics & 5 \\
\hline 9 & Journal of Neurology Neurosurgery and & 4 \\
\hline 9 & Psychiatry & 4 \\
\hline 11 & Journal of Neuroscience & 3 \\
\hline 12 & Archives of Neurology & 2 \\
\hline
\end{tabular}

GGE, genetic generalized epilepsy.

Table 3. Countries of origin of the top-100 cited articles in the field of GGE

\begin{tabular}{lcc}
\hline Rank & Country & Number of articles \\
\hline 1 & USA & 31 \\
\hline 2 & UK & 14 \\
3 & Germany & 11 \\
\hline 3 & Australia & 11 \\
\hline 5 & France & 7 \\
\hline 6 & Canada & 9 \\
\hline 7 & Saudi Arabia & 3 \\
\hline 8 & Italy & 2 \\
\hline 8 & Brazil & 2 \\
\hline 8 & Netherlands & 2 \\
\hline 8 & China & 2 \\
\hline 12 & Switzerland & 1 \\
\hline 12 & New Zealand & 1 \\
\hline 12 & South Korea & 1 \\
\hline 12 & Sweden & 1 \\
12 & Israel & 1 \\
12 & Japan & 1 \\
\hline
\end{tabular}

GGE, genetic generalized epilepsy.
Table 4. Originating institutions with at least 2 of the top-100 cited articles in the field of GGE

\begin{tabular}{lcc}
\hline Rank & Institution & $\begin{array}{c}\text { Number } \\
\text { of articles }\end{array}$ \\
\hline 1 & University of Melbourne & 9 \\
\hline 2 & University of California at Los Angeles & 7 \\
\hline 3 & University College London & 6 \\
\hline 4 & University of McGill & 5 \\
\hline 5 & University of New York & 4 \\
\hline 6 & University of King Khalid & 3 \\
\hline 6 & University of Humboldt & 3 \\
\hline 6 & King's College London & 3 \\
\hline 9 & University of Saint Paul & 2 \\
\hline 9 & University of Calgary & 2 \\
\hline 9 & University of Cincinnati & 2 \\
\hline 9 & University of Virginia & 2 \\
\hline 9 & University of Wake Forest & 2 \\
\hline 9 & University of Vanderbilt & 2 \\
\hline GGE, genetic generalized epilepsy. & \\
\hline
\end{tabular}

Table 5. First authors with at least 2 of the top-100 cited articles in the field of GGE

\begin{tabular}{lcc}
\hline Rank & First author & Number of articles \\
\hline 1 & Panayiotopoulos CP & 3 \\
2 & Dumer M & 2 \\
2 & Gelisse P & 2 \\
\hline 2 & Glauser TA & 2 \\
2 & Greengerg DA & 2 \\
\hline 2 & Grunewald RA & 2 \\
\hline 2 & Janz D & 2 \\
\hline 2 & Kang JQ & 2 \\
2 & Khosravani H & 2 \\
2 & Marini C & 2 \\
\hline 2 & Vitko I & 2 \\
\hline 2 & Woermann FG & 2 \\
\hline
\end{tabular}

GGE, genetic generalized epilepsy. 
25

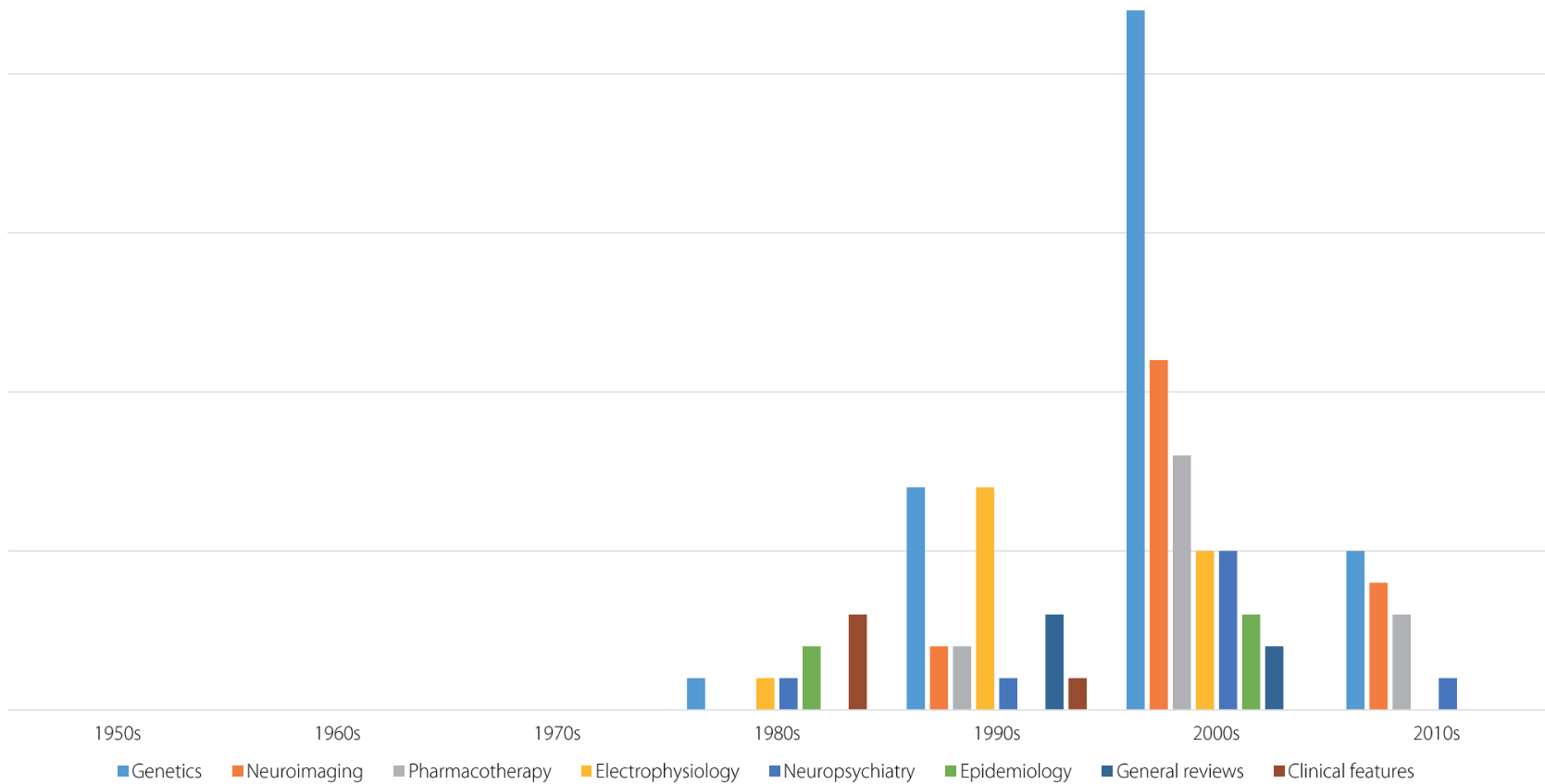

Fig. 1. Number of publications with the top-100 cited articles in the field of genetic generalized epilepsy.

\section{DISCUSSION}

This study identified and characterized the top-100 cited articles in the field of GGE. These citation classics may enable the identification of seminal advances in GGE and provide a historical perspective on the scientific progress of the field of epilepsy.

The top-ranked article had a title of "Mutant gamma-aminobutyric acid receptor subtype $A(G A B A)(A)$ receptor gamma2-subunit in childhood absence epilepsy and febrile seizure," its first author was Wallace, it was published in Nature Genetics, and it has been cited 580 times. ${ }^{13}$ The authors found a mutation in a gene encoding a GABA(A) receptor subunit in a large family with epilepsy, and the two main phenotypes were childhood absence epilepsy and febrile seizures. ${ }^{13}$ The second-ranked article was also published in Nature Genetics, and reported that an Ala322Asp mutation in the GABRA1 gene that encodes the alpha1 subunit of GABA(A) was found in affected individuals of a large French Canadian family with juvenile myoclonic epilepsy. ${ }^{14}$ Both of these articles reported on genetic studies. The third-ranked article was a review article on childhood absence epilepsy by Crunelli V that appeared in Nature Reviews Neuroscience.

Citation analysis can identify emerging topics and the relevant trends in a particular field., ${ }^{5,6}$ The present study found that genetics was the most-common topic in the top-100 cited articles on GGE, followed by neuroimaging. Genetics and neuroimaging were the most-common topics for each decade from the 1990s to the 2010s. Since genetic epilepsy directly results from a known or presumed genetic mutation whose core symptom is seizures, ${ }^{1}$ it is not surprising that genetics was the most-common topic in the field of GGE. In addition, due to the introduction and advent of next-generation sequencing and genome-wide association studies, the development and application of high-throughput genetic testing has resulted in the discovery of hundreds of epilepsy-associated genes. ${ }^{15}$ Thus, we can assume that the number of articles on genetics will increase in the future.

The application of neuroimaging in epilepsy has also increased rapidly and evolved thanks to the substantial advancements in image-analysis techniques in recent decades. ${ }^{16}$ Early studies involving brain magnetic resonance 
imaging (MRI) did not reveal abnormalities in patients with GGE. However, more-recent voxel-based morphometry and structural/functional connectivity studies based on diffusion-tensor imaging and functional MRI have revealed abnormal morphologies and networks of the brain in GGE. ${ }^{17-}$ ${ }^{20}$ These developments are associated with increasing numbers of related articles being published in scientific journals that could have a great impact on GGE.

The topics addressed in the citation classics varied among the decades, and we discovered some interesting trends in the topics over time. We noted that the most-cited articles on GGE were published during the 2000s. This contrasts with most bibliometric analyses on other topics demonstrating that the most-cited articles are published during the 1990s. ${ }^{7-}$ 11,21 Thus, we can infer that there have been considerable developments in research on GGE in recent years, which might be attributable to recent developments in research techniques such as genetics and neuroimaging in this field.

We also found that the most-cited articles were published in Epilepsia, which is the official publication of the ILAE. This is perhaps related to the epilepsy-specific journals with high impact factors being focused on GGE. Moreover, we found that about one-third of the 100 top-cited articles originated from institutions in the USA, reflecting the huge influence of the USA in health science research in general, which is probably due to both the large size of the American scientific community and its high research budget. ${ }^{22-25}$ However, a citation analysis in the field of neurology found that from half to two-thirds of the articles originated from the USA. In addition, we found that 20 articles reported on studies performed in Asia and Oceania (12 and 8 articles, respectively), which was a prominently higher ratio than in other citation analyses. We can assume that this finding is associated with the most-cited articles on GGE being published during the 2000s. Recently there have been increasing numbers of articles originating from Asia and Oceania in the field of neurology research, especially from China. ${ }^{26}$

This study is the first to perform a citation analysis of GGE. The findings could be used to identify recent advances in the field of GGE, provide a historical perspective of its scientific progress, and be used for education purposes. However, there were several inherent limitations in the research methodology. There is ongoing debate about the value of citation rates. A naive argument is that an article of great- er value will be cited more often. ${ }^{27}$ However, the number of citations could be influenced by factors other than the quality and originality of the reported research, such as the characteristics of the involved researchers, institutions, and funding agencies. ${ }^{21}$ Furthermore, analyzing the total number of citations favors older articles. ${ }^{28}$ The citation frequency of a scientific article is typically associated with a time delay of $1-2$ years after its publication. ${ }^{29}$ This interval will bias evaluations of the rank and significance of recent publications. However, the use of citation rates is still widely accepted as the best method for judging the impact of the articles, with the impact factor considered indicative of the quality and rank of a given journal in its specific field of interest. ${ }^{30}$

This study has identified the top-100 cited articles on GGE. The identified citation classics represent landmark articles on GGE, and they provide useful insights into international research leaders and the research trends in the field.

\section{Author Contributions}

Conceptualization and data analysis: Kang Min Park. Methodology: Seongho Park, Dongah Lee. Original draft preparation: Bong Soo Park.

\section{Conflict of Interest}

None of the authors has any conflict of interest to disclose.

\section{REFERENCES}

1. Scheffer IE, Berkovic S, Capovilla G, Connolly MB, French J, Guilhoto $L$, et al. ILAE classification of the epilepsies: position paper of the ILAE Commission for Classification and Terminology. Epilepsia 2017;58:512-521.

2. Berg AT, Berkovic SF, Brodie MJ, Buchhalter J, Cross JH, van Emde Boas W, et al. Revised terminology and concepts for organization of seizures and epilepsies: report of the ILAE Commission on Classification and Terminology, 2005-2009. Epilepsia 2010;51:676685.

3. Jallon P, Latour P. Epidemiology of idiopathic generalized epilepsies. Epilepsia 2005;46 Suppl 9:10-14.

4. Andersen J, Belmont J, Cho CT. Journal impact factor in the era of expanding literature. J Microbiol Immunol Infect 2006;39:436443.

5. Garfield E, Cawkell AE. Citation analysis studies. Science 
1975;189:397.

6. Garfield E. Citation analysis as a tool in journal evaluation. Science 1972;178:471-479.

7. Kim Y, Yoon DY, Kim JE, Park KM, Lee JH, Song HK, et al. Citation classics in stroke: the top-100 cited articles on hemorrhagic stroke. Eur Neurol 2017;78:210-216.

8. Park KM, Park BS, Park S, Yoon DY, Bae JS. Top-100 cited articles on headache disorders: a bibliometric analysis. Clin Neurol Neurosurg 2017;157:40-45.

9. Kim JE, Park KM, Kim Y, Yoon DY, Bae JS. Citation classics in central nervous system inflammatory demyelinating disease. Brain Behav 2017;7:e00700.

10. Kim JE, Kim JK, Park KM, Kim Y, Yoon DY, Bae JS. Top-100 cited articles on Guillain-Barré syndrome: a bibliometric analysis. J Peripher Nerv Syst 2016;21:329-338.

11. Park KM, Kim SE, Lee Bl, Kim HC, Yoon DY, Song HK, et al. Top 100 cited articles on epilepsy and status epilepticus: a bibliometric analysis. J Clin Neurosci 2017;42:12-18.

12. Park KM, Kim JE, Kim Y, Kim SE, Yoon DY, Bae JS. Searching the footprints of pioneers on neurology: a bibliometric analysis. Eur Neurol 2017;77:152-161.

13. Wallace RH, Marini C, Petrou S, Harkin LA, Bowser DN, Panchal RG, et al. Mutant GABA(A) receptor gamma2-subunit in childhood absence epilepsy and febrile seizures. Nat Genet 2001;28:49-52.

14. Cossette $\mathrm{P}$, Liu L, Brisebois K, Dong H, Lortie A, Vanasse M, et al. Mutation of GABRA1 in an autosomal dominant form of juvenile myoclonic epilepsy. Nat Genet 2002;31:184-189.

15. Symonds JD, McTague A. Epilepsy and developmental disorders: next generation sequencing in the clinic. Eur J Paediatr Neurol 2020;24:15-23.

16. Hoeffner EG, Mukherji SK, Srinivasan A, Quint DJ. Neuroradiology back to the future: brain imaging. AJNR Am J Neuroradiol 2012;33:5-11.

17. Kim JH, Lee JK, Koh SB, Lee SA, Lee JM, Kim SI, et al. Regional grey matter abnormalities in juvenile myoclonic epilepsy: a vox- el-based morphometry study. Neuroimage 2007;37:1132-1137.

18. Lee HJ, Seo SA, Lee BI, Kim SE, Park KM. Thalamic nuclei volumes and network in juvenile myoclonic epilepsy. Acta Neurol Scand 2020;141:271-278.

19. Park KM, Kim TH, Han YH, Mun CW, Shin KJ, Ha SY, et al. Brain morphology in juvenile myoclonic epilepsy and absence seizures. Acta Neurol Scand 2016;133:111-118.

20. Lee HJ, Park KM. Structural and functional connectivity in newly diagnosed juvenile myoclonic epilepsy. Acta Neurol Scand 2019;139:469-475.

21. Patience GS, Patience CA, Blais B, Bertrand F. Citation analysis of scientific categories. Heliyon 2017;3:e00300

22. Paladugu R, Schein M, Gardezi S, Wise L. One hundred citation classics in general surgical journals. World J Surg 2002;26:10991105.

23. Loonen MP, Hage JJ, Kon M. Plastic Surgery Classics: characteristics of 50 top-cited articles in four Plastic Surgery Journals since 1946. Plast Reconstr Surg 2008;121:320e-327e.

24. Baltussen A, Kindler $\mathrm{CH}$. Citation classics in anesthetic journals. Anesth Analg 2004;98:443-451.

25. Tsai YL, Lee CC, Chen SC, Yen ZS. Top-cited articles in emergency medicine. Am J Emerg Med 2006;24:647-654.

26. Jamieson PW. Neurology research: a journal survey, 1980-1990. Ann Neurol 1992;32:87-93.

27. Smith R. Beware the tyranny of impact factors. J Bone Joint Surg Br 2008;90:125-126

28. Pepe A, Kurtz MJ. A measure of total research impact independent of time and discipline. PLoS One 2012;7:e46428.

29. Callaham M, Wears RL, Weber E. Journal prestige, publication bias, and other characteristics associated with citation of published studies in peer-reviewed journals. JAMA 2002;287:28472850.

30. Seglen PO. Citations and journal impact factors: questionable indicators of research quality. Allergy 1997;52:1050-1056. 